\title{
Real-life events in multimedia: detection, representation, retrieval, and applications
}

\author{
Vasileios Mezaris • Ansgar Scherp • \\ Ramesh Jain • Mohan S. Kankanhalli
}

Published online: 4 April 2013

(C) Springer Science+Business Media New York 2013

The multimedia content that all of us frequently capture with our different multimedia enabled devices (e.g. cameras, smartphones, tablets) is typically the digital residue of a real-life event that unfolded before us, such as a graduation, a trip, a football game, or a natural disaster; it is this real-life event that we try to immortalize through the digital content creation process. Similarly, at an organizational (as opposed to personal) scale, multimedia content such as satellite images and radar signals are often captured in order to document natural or man-induced real-life events, such as weather phenomena or sea pollution incidents. Despite the central role that real-life events play in the generation and also the later interpretation of multimedia content, though, (since, for instance, what is the meaning and the value of a picture of a football field with some players on it, unless we can put it in

\footnotetext{
A. Scherp $(\varangle)$

Institute of Computer Science and Business Informatics,

University of Mannheim, B6, 26, 68131 Mannheim, Germany

e-mail: ansgar@informatik.uni-mannheim.de

V. Mezaris

Information Technologies Institute (ITI), Centre for Research and Technology Hellas (CERTH), 6th Km. Charilaou-Thermi Road, P.O. Box 60361,

57001 Thermi-Thessaloniki, Greece

M. S. Kankanhalli

Department of Computer Science, School of Computing, National University of Singapore,

117417 Singapore, Singapore

R. Jain

University of California, Irvine, CA 92697, USA
} 
event-context: which football game was that? And, what exactly happened in that game that makes it special?) the organization and retrieval of multimedia content are yet to fully embrace event-centric methodologies.

In response to this challenge, this special issue focuses on methods for the eventbased processing and organization of multimedia content, with particular emphasis on the detection of real-live events in multimedia, the modeling of such real-life events, the sharing and the event-based retrieval of content, and the development of novel applications that jointly consider multimedia content and the real-life events that this content represents. The special issue includes nine papers that highlight different problems and solutions in these domains.

In the first paper, Scherp and Mezaris focus on the representation of events using suitable models. In particular, they conduct a survey of existing event models, and attempt to compare these models along a number of different dimensions that an event model should cover ("event aspects"). Further to this, they analyze how the different aspects of events relate to each other and how they can be applied together, and conclude with some references and thoughts on how the linking between the multimedia data and the events can be achieved, so as to provide the basis for future event-based multimedia applications.

The next three papers deal with event detection and event-based organization for still images. Dao, Dang-Nguyen and De Natale address the problem of associating personal image collections with events by analyzing the photo collection of an event as a whole, rather than looking at individual images. The objective is to detect event-types such as graduation, wedding, or different types of vacations and sports events, which describe the collection. In order to create a composite event signature for an image collection, they combine Saliency, Gist and Time information and introduce the notions of Gist-Saliency Signature Image Base (GS-SIB), which captures dominant colors and saliency information for all the images belonging to a photo collection, and Temporal SIB (T-SIB), which captures the temporal evolution of the images.

Ruocco and Ramampiaro look at the problem of event-based organization of images that are available in online photo-sharing applications such as Flickr. They propose a clustering approach, which takes into account textual annotations as well time and geo-location metadata of the images. To this end they extend the wellknown Suffix Tree Clustering (STC) algorithm, originally developed for clustering text documents. They also investigate how the processing of the images at different time and space granularities affects their event-based organization.

Zigkolis, Papadopoulos, Filippou, Kompatsiaris and Vakali take the work on event-based organization of online images one step further, presenting a semiautomatic tool for the user-assisted generation of ground-truth image-event associations from online image collections. Specifically, they present CrEve, a collaborative event annotation framework which facilitates the annotation process and increases the coverage of the generated ground truth. Furthermore, the paper discusses the results of a user study that quantifies the contribution of different event dimensions in the event annotation process, confirming, for instance, the prevalence of spatiotemporal queries as the prime option of discovering event-related content in a large collection. 
The next four papers deal with event detection and event-based organization of video content, with the latter content ranging from broad-domain user-generated videos to content captured under well-controlled conditions for supporting very specific applications. Cricri, Dabov, Curcio, Mate and Gabbouj exploit the readings of auxiliary sensors such as accelerometers and GPS receivers, which are typically included in camera-enabled devices, for detecting interesting events in user generated videos and for extracting high-level contextual information about the recording activity. In addition, they exploit multiple audio-visual recordings of a common event (e.g., music concerts), when available, to extract additional event-related information such as regions of interest in the videos.

Poulisse, Patsis and Moens propose a method for identifying the semantic structure in long semi-structured video streams. They identify chains, i.e., local clusters of audio or visual features that are repeated in time, and use each chain as an indicator that the temporal interval it demarcates is part of a single semantic event. By layering all the chains over each other, dense regions emerge from the overlapping chains, revealing the semantic structure of the video.

The paper by Cheng, Liu, Zhao, Ye and Sun addresses the problem of detecting events in the daily activities of seniors within their home, for the purpose of monitoring the seniors' health. The authors introduce as part of their system a subspace Naive-Bayesian Mutual Information Maximization (sNBMIM) algorithm, which divides the feature space into a number of subspaces and allows the kernel and normalization parameters to vary between different subspaces. The presented senior home activity recognition system is evaluated for eight categories of everyday home events, such as sleep, eat, wash.

Spampinato, Palazzo, Boom et al. look at an environment-related application, and specifically present a system for understanding fish behavior during typhoon events by analyzing underwater video footage. The first step of the system that they propose involves the detection of "typhoon" events from the video recordings, which is based on video texture analysis and classification. Then, for understanding fish behavior during a typhoon event, they perform trajectory extraction and clustering from the video clip. They evaluate their approach on a set of underwater videos captured as part of the Fish4Knowledge EU project.

The special issue concludes with another paper on the detection of environmentrelated events. Talukder and Panangadan present an event-based multimedia processing framework for the detection, retrieval, and cross-media content assimilation of geo-spatiotemporal phenomena such as cyclones. They draw their primary content from various sources, such as remote satellites, in-situ visual sensors and weather bulletins, and use it for detecting the geo-spatiotemporal events of interest. Subsequently, they derive appropriate descriptions of the detected events, and use them for assimilating further event information across different media sources from the web.

In this special issue, we tried to include a rich set of works on the processing and organization of multimedia content according to real-life events. We hope that you will enjoy reading them. At this point, we would also like to take the opportunity to thank all the contributors of this special issue and all the reviewers who helped us in composing it. 


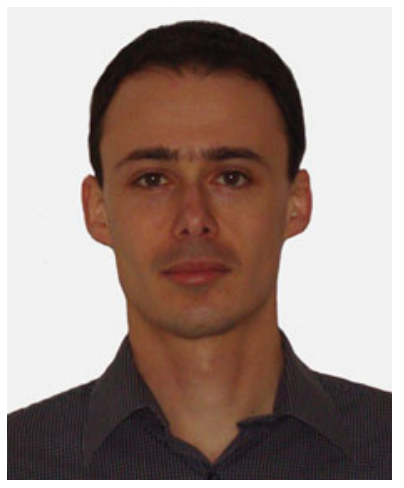

Vasileios Mezaris received the Diploma and the Ph.D. degree in Electrical and Computer Engineering from the Aristotle University of Thessaloniki, Thessaloniki, Greece, in 2001 and 2005, respectively. He is a Senior Researcher (Researcher C) with the Information Technologies Institute / Centre for Research and Technology Hellas, Thessaloniki, Greece. His research interests include image and video analysis, event detection in multimedia, machine learning for multimedia analysis, content-based and semantic image and video retrieval, medical image analysis. He is the co-author of 24 papers in refereed international journals, 9 book chapters, two patents and more than 80 papers in international conferences. He currently serves as an Associate Editor for the IEEE Transactions on Multimedia. He is a member of the IEEE.

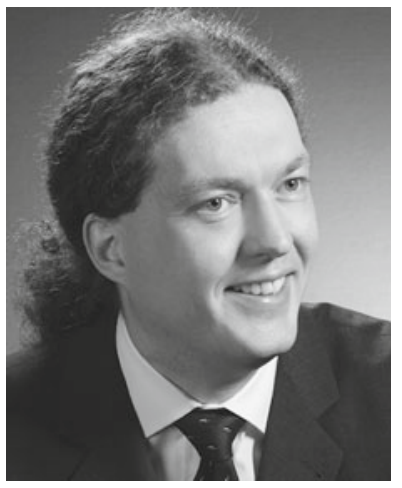

Ansgar Scherp is Junior professor for Media Informatics and New Media in Business Informatics at the Research Group on Data and Web Science of the University of Mannheim since August 2012. Prior to that he was working as Junior professor for Semantic Web at the University of KoblenzLandau in the Institute for Information Systems Research since April 2011 and lead the focus group on Interactive and Multimedia Web at the Institute for Web Science and Technologies (WeST) at the same university since May 2008. He has studied computer science at the University of Oldenburg, Germany and has received the Advancement Award for Outstanding Results in Studies from the Association for Electrical, Electronic \& Information Technologies (VDE), Germany in 1998. He 
finished his $\mathrm{PhD}$ with the thesis title "A Component Framework for Personalized Multimedia Applications" at the University of Oldenburg, Germany with distinction in 2006. Afterwards, Mr. Scherp has been EU Marie Curie Fellow with Prof. Ramesh Jain at the Donald Bren School of Information and Computer Sciences, University of California, Irvine, USA in Los Angeles between November 2006 to October 2007. He has lead the University of Koblenz-Landau's activities in the EU Integrated Project WeKnowIt from 2008 to 2011. Here, he has been leading the work packages on knowledge management and mass intelligence and has been member of the project management board and steering board committee. Mr. Scherp is scientific leader of the EU project SocialSensor, where the University of Koblenz-Landau is leading the work package on user modeling and presentation. In December 2011, he has received his Venia Legendi (Habilitation) with the thesis title "Semantic Media Management: Process Innovation along the Value Chain of Media Companies" (in German) from the University of Koblenz-Landau, Germany.

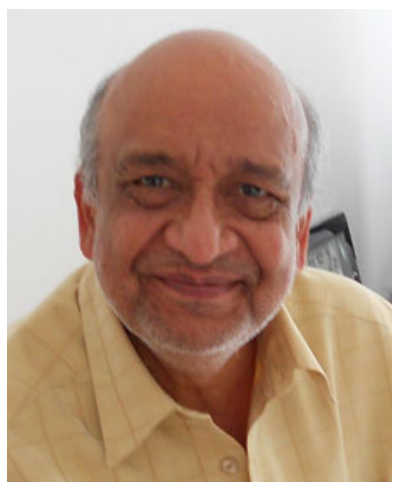

Ramesh Jain is an entrepreneur, researcher, and educator. He is a Donald Bren Professor in Information \& Computer Sciences at University of California, Irvine where he is doing research in EventWeb and Experiential Computing for developing and building Social Life Networks. Earlier he served on faculty of Georgia Tech, University of California at San Diego, The university of Michigan, Ann Arbor, Wayne State University, and Indian Institute of Technology, Kharagpur. He has been an active member of professional community serving in various positions and contributing more than 350 research papers. He is the recipient of several awards including the ACM SIGMM Technical Achievement Award 2010. He is a Fellow of ACM, IEEE, AAAI, IAPR, and SPIE. Ramesh co-founded several companies, managed them in initial stages, and then turned them over to professional management. 


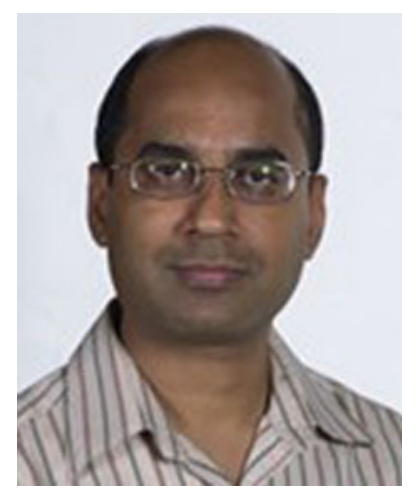

Mohan S. Kankanhalli is a Professor at the Department of Computer Science of the National University of Singapore. He is also the Associate Provost for Graduate Education at NUS. Mohan obtained his BTech (Electrical Engineering) from the Indian Institute of Technology, Kharagpur, in 1986 and his MS and PhD (Computer and Systems Engineering) from the Rensselaer Polytechnic Institute in 1998 and 1990, respectively. He is actively involved in organizing of many major conferences in the area of Multimedia. He is on the editorial boards of several journals including the ACM Transactions on Multimedia Computing, Communications, and Applications, Springer Multimedia Systems Journal, Pattern Recognition Journal and Multimedia Tools \& Applications. His current research interests are in Multimedia Systems, Digital Video Processing and Multimedia Security (surveillance, digital rights management and privacy). 